\title{
Paper WannaCry Ransomware: Analysis of Infection, Persistence, Recovery Prevention and Propagation Mechanisms
}

\author{
Maxat Akbanov ${ }^{1}$, Vassilios G. Vassilakis ${ }^{2}$, and Michael D. Logothetis ${ }^{3}$ \\ ${ }^{1}$ Department of Computer Science, University of York, York, United Kingdom \\ ${ }^{2}$ University of York, York, United Kingdom \\ ${ }^{3}$ WCL, Dept. of Electrical and Computer Engineering, University of Patras, Patras, Greece
}

https://doi.org/10.26636/jtit.2019.130218

\begin{abstract}
In recent years, we have been experiencing fast proliferation of different types of ransomware targeting home users, companies and even critical telecommunications infrastructure elements. Modern day ransomware relies on sophisticated infection, persistence and recovery prevention mechanisms. Some recent examples that received significant attention include WannaCry, Petya and BadRabbit. To design and develop appropriate defense mechanisms, it is important to understand the characteristics and the behavior of different types of ransomware. Dynamic analysis techniques are typically used to achieve that purpose, where the malicious binaries are executed in a controlled environment and are then observed. In this work, the dynamic analysis results focusing on the infamous WannaCry ransomware are presented. In particular, WannaCry is examined, during its execution in a purpose-built virtual lab environment, in order to analyze its infection, persistence, recovery prevention and propagation mechanisms. The results obtained may be used for developing appropriate detection and defense solutions for WannaCry and other ransomware families that exhibit similar behaviors.
\end{abstract}

Keywords—dynamic malware analysis, ransomware, WannaCry.

\section{Introduction}

Ransomware threat is currently considered to be the main moneymaking scheme for cyber criminals and the key threat to Internet users [1], [2]. In recent years, the appearance of new types of ransomware has been observed, combining the use of worm-like spreading mechanisms and advanced recovery prevention schemes. Recent examples include WannaCry [3], [4] and Petya [5], [6], which exploit the weaknesses of Microsoft Windows, as well as BadRabbit [7], which spreads via insecure compromised websites.

From the defense perspective, the design of new countermeasures is considered, in addition to traditional security approaches, an important and trending task in this field. Such a design, however, requires a comprehensive analysis of ransomware functionality and behavior. This typically involves a wide range of malware analysis tools and tech- niques. Such techniques may be broadly classified as static and dynamic. Static analysis is performed without executing the malicious binary, while dynamic analysis involves executing the binary in an isolated environment.

In one of our previous works [8], we performed an initial static and dynamic analysis of WannaCry to identify its resources and functions, as well as its use of dynamic-link libraries (DLLs) and communication protocols. In this work, we have performed a comprehensive dynamic analysis, focusing on WannaCry's infection, persistence, recovery prevention and propagation mechanisms. The techniques presented are also applicable in the cases of other ransomware families whose characteristics are similar to that of WannaCry, such as worm-spreading mechanisms and public-key based encryption. In particular, the research presented examines WannaCry's behavior during its execution in a safe, purpose-built virtual lab environment at the University of York. The results obtained may form a basis for designing and developing effective ransomware defense solutions.

The rest of the paper is organized as follows. In Section 2, we present the relevant background information on ransomware in general and on WannaCry in particular. In Section 3, the main findings from the dynamic analysis of WannaCry we have performed, including its encryption process, recovery prevention and propagation mechanisms, are presented. Finally, Section 4 draws conclusions and discusses potential future directions.

\section{Background}

\subsection{Ransomware}

Ransomware is a type of malicious software (malware) that prevents users from accessing or limits their access to the system or files, either by locking the screen or by encrypting files, until a ransom is paid [9]. In most cases, ransomware leaves the user with very few options, such as only allowing the victim to communicate with the attacker and pay the ransom. 
The most common types of ransomware use some form of encryption, including both symmetric and public-key based encryption schemes. Ransomware that relies on publickey encryption is particularly difficult to mitigate, since the encryption keys are stored in a remote command and control $(\mathrm{C} \& \mathrm{C})$ server. There is usually a time limit for ransom to be paid, the users are provided with a special website to purchase cryptocurrency (e.g. Bitcoins) and stepby-step instructions on how to pay the ransom.

The lifecycle of modern day ransomware typically consists of the following steps [10]: distribution, infection, C\&C communications, file search, file encryption and ransom demand.

\subsection{WannaCry}

WannaCry ransomware (also known as Wana Decrypt0r, WCry, WannaCry, WannaCrypt, and WanaCrypt0r) was observed during a massive attack across multiple countries on 12 May 2017 [11]. According to multiple reports from security vendors, the total of 300,000 systems in over 150 countries had been severely damaged. The attack affected a wide range of sectors, including healthcare, government, telecommunications and gas/oil production.

The difficulty in protecting against WannaCry stems from its ability to spread to other systems by using a worm component. This feature makes the attacks more effective and requires defense mechanisms that can react quickly and in real time. Furthermore, WannaCry has an encryption component that is based on public-key cryptography.

During the infection phase, WannaCry uses the EternalBlue and DoublePulsar exploits that were allegedly leaked in April 2017 by a group called The Shadow Brokers. EternalBlue exploits the server message block (SMB) vulnerability that was patched by Microsoft on March 14, 2017 and has been described in the security bulletin MS17-010 [12]. This vulnerability allows the adversaries to execute a remote code on the infected machines by sending specially crafted messages to an SMB v1 server, connecting to TCP ports 139 and 445 of unpatched Windows systems. In particular, this vulnerability affects all unpatched Windows versions starting from Windows XP to Windows 8.1, except for Windows 10 .

DoublePulsar is a persistent backdoor that may be used to access and execute code on previously compromised systems, thus allowing the attackers to install additional malware on the system. During the distribution process, WannaCry's worm component uses EternalBlue for initial infection through the SMB vulnerability, by actively probing appropriate TCP ports and, if successful, tries to implant the DoublePulsar backdoor on the infected systems.

\section{WannaCry Analysis}

In this section, we present our findings based on the dynamic analysis of WannaCry we have performed. Samples of WannaCry were obtained from VirusShare [13]. Two executable files were analyzed: the worm component and the encryption component (Table 1).

Table 1

WannaCry components

\begin{tabular}{|c|l|}
\hline & \multicolumn{1}{|c|}{ Worm component } \\
\hline \hline MD5 & db349b97c37d22f5ea1d1841e3c89eb4 \\
\hline SHA1 & $\begin{array}{l}\text { e889544aff85ffaf8b0d0da705105dee7c } \\
\text { 97fe26 }\end{array}$ \\
\hline SHA256 & $\begin{array}{l}\text { 24d004a104d4d54034dbcffc2a4b19a11 } \\
\text { f39008a575aa614ea04703480b1022c }\end{array}$ \\
\hline File type & $\begin{array}{l}\text { PE32 executable (GUI) Intel 80386, } \\
\text { for MS Windows }\end{array}$ \\
\hline \hline MD5 & \multicolumn{1}{|c|}{ Encryption component } \\
\hline SHA1 & $\begin{array}{l}\text { 5ff4653afaabcbf0150d1a3ab2c2e74f3a4 } \\
\text { 426467 }\end{array}$ \\
\hline SHA256 & $\begin{array}{l}\text { ed01ebfbc9eb5bbea545af4d01bf5f1071 } \\
\text { 661840480439c6e5babe8e080e41aa }\end{array}$ \\
\hline File type & $\begin{array}{l}\text { PE32 executable (GUI) Intel 80386, } \\
\text { for MS Windows }\end{array}$ \\
\hline
\end{tabular}

\subsection{Testbed}

In order to analyze WannaCry, a virtual testbed shown in Fig. 1 was built. The characteristics of the host machine are as follows: Intel Core i7-4700MQ $2.40 \mathrm{GHz}$ and $16 \mathrm{~GB}$ RAM. The host machine acts as a virtual switch and is running REMnux [14], which is a free Linux toolkit for reverse engineering and malware analysis. Two virtual machines (VMs), running Windows 7 SP1, were used. The first VM was infected with WannaCry, whereas the other VM was clean. A custom network VMnet 5 - 192.168.180.0/24 was created with the Virtual Network Editor option in VMWare hypervisor. This testbed allows observing domain name system (DNS) queries made by WannaCry during the infection and replication process across internal and external

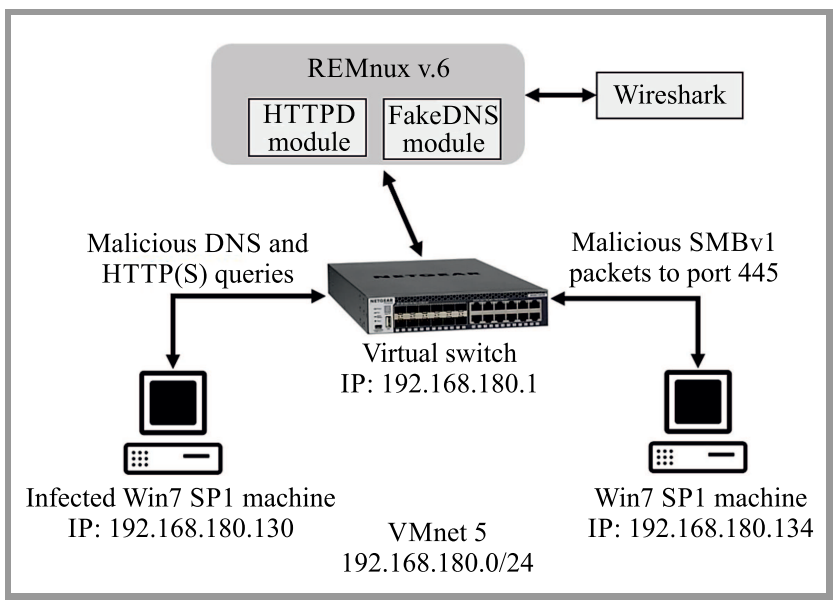

Fig. 1. Testbed for dynamic WannaCry analysis. 
networks via port 445 of the SMB v1 protocol. The REMnux machine acts as a DNS and HTTP server, and is able to intercept all network communications using Wireshark. DNS and HTTP services in REMnux were enabled using FakeDNS and HTTP Daemon utilities, respectively.

The system level actions performed by WannaCry were observed on the infected Windows 7 SP1 machine with the 192.168.180.130 IP address. In order to observe and report the actions that WannaCry took while running on the system, the SysAnalyzer tool [15] was used. The main benefit of SysAnalyzer is that it is capable of taking system snapshots before and after malware execution, thus making it possible to inspect system attributes, such as running processes, open ports, DLLs loaded, registry key changes, run time file modifications, scheduled tasks, mutual exclusion objects (mutexes) and network connections. SysAnalyzer is also capable of taking memory dumps and scanning them for specific regular expressions. Before executing the WannaCry sample on the infected machine, the SysAnalyzer's configuration wizard was set to apply a 120 s delay between system snapshots, thus allowing to inspect all system attribute changes.

\subsection{Libraries and Functions}

Analysis performed with the Pestudio tool [16] revealed that the worm and the encryption components of WannaCry

Table 2

DLLs of the worm component

\begin{tabular}{|l|c|l|}
\hline \multicolumn{1}{|c|}{ Library } & Imports & \multicolumn{1}{c|}{ Description } \\
\hline \hline ws2_32.dll & 13 & $\begin{array}{l}\text { Windows Socket 2.0 32-bit } \\
\text { DLL }\end{array}$ \\
\hline iphlpapi.dll & 2 & IP Helper API \\
\hline wininet.dll & 3 & Internet Extensions for Win32 \\
\hline kernel32.dll & 32 & $\begin{array}{l}\text { Windows NT Base API } \\
\text { Client DLL }\end{array}$ \\
\hline advapi32.dll & 11 & $\begin{array}{l}\text { Advanced Windows 32 Base } \\
\text { API }\end{array}$ \\
\hline msvcp60.dll & 2 & $\begin{array}{l}\text { Windows NT C++ Runtime } \\
\text { Library DLL }\end{array}$ \\
\hline msvcrt.dll & 28 & Windows NT CRT DLL \\
\hline
\end{tabular}

Table 3

DLLs of the encryption component

\begin{tabular}{|c|c|l|}
\hline \multicolumn{1}{|c|}{ Library } & Imports & \multicolumn{1}{c|}{ Description } \\
\hline \hline kernel32.dll & 54 & $\begin{array}{l}\text { Windows NT Base API } \\
\text { Client DLL }\end{array}$ \\
\hline advapi32.dll & 10 & $\begin{array}{l}\text { Advanced Windows 32 Base } \\
\text { API }\end{array}$ \\
\hline user32.dll & 1 & $\begin{array}{l}\text { Multi-User Windows User } \\
\text { API Client DLL }\end{array}$ \\
\hline msvcrt.dll & 49 & Windows NT CRT DLL \\
\hline
\end{tabular}

contain DLLs shown in Tables 2 and 3, respectively. During its execution, the worm component invokes iphlpapi.dll to retrieve network configuration settings for the infected host. Kernel32.dll and msvcrt.dll are the two libraries most frequently invoked by the encryption component. This may indicate that the main encryption functionality was implemented by these two malicious libraries. To confirm this, the imported functions of the libraries needed to be examined.

Table 4

Functions of the encryption component

\begin{tabular}{|l|c|}
\hline \multicolumn{1}{|c|}{ Function } & Location \\
\hline \hline GetCurrentThread & 0xa53a \\
\hline GetStartupInfoA & 0xa97a \\
\hline StartServiceCtrDispatcherA & 0xa6f6 \\
\hline RegisterServiceCtrDispatcherA & 0xa6d8 \\
\hline CreateServiceA & 0xa688 \\
\hline StartServiceA & 0xa662 \\
\hline CryptGenRandom & 0xa650 \\
\hline CryptAcquireContextA & 0xa638 \\
\hline OpenServiceA & 0xa714 \\
\hline GetAdaptersInfo & 0xa792 \\
\hline InternetOpenUrlA & 0xa7c8 \\
\hline
\end{tabular}

Table 5

Functions of the encryption component

\begin{tabular}{|l|l|}
\hline \multicolumn{1}{|c|}{ Function } & Location \\
\hline \hline OpenMutexA & 0xda84 \\
\hline GetComputerNameW & 0xd8b2 \\
\hline CreateServiceA & 0xdc2a \\
\hline OpenServiceA & 0xdc62 \\
\hline StartServiceA & 0xdc52 \\
\hline CryptReleaseContext & 0xdc14 \\
\hline RegCreateKeyW & 0xdc04 \\
\hline fopen & 0xdcd4 \\
\hline fread & 0xdccc \\
\hline fwrite & 0xdcc2 \\
\hline fclose & 0xdcb8 \\
\hline CreateFileA & 0xd922 \\
\hline ReadFile & 0xd964 \\
\hline
\end{tabular}

The imported functions of the samples were observed by Pestudio. The most suspicious functions identified among them are shown in Tables 4 and 5. One may observe that in general, WannaCry uses Microsoft's crypto, file management and $\mathrm{C}$ runtime file APIs. The crypto API library is used to generate and manage random symmetric and asymmetric cryptographic keys. 
root@remnux: \# fakedns 192.168 .180 .128

pyminifakeDNS:: dom.query. 60 IN A 192.168.180.128

Respuesta: watson.microsoft.com. $\rightarrow>192.168 .180 .128$

Respuesta: teredo.ipv6.microsoft.com. $\rightarrow 192.168 .180 .128$

Respuesta: ww. iuaerfsodngifianosdfihaosuriifaewrwerawea.com. -> 192.168.180.128

Fig. 2. FakeDNS capture of the malicious DNS request.

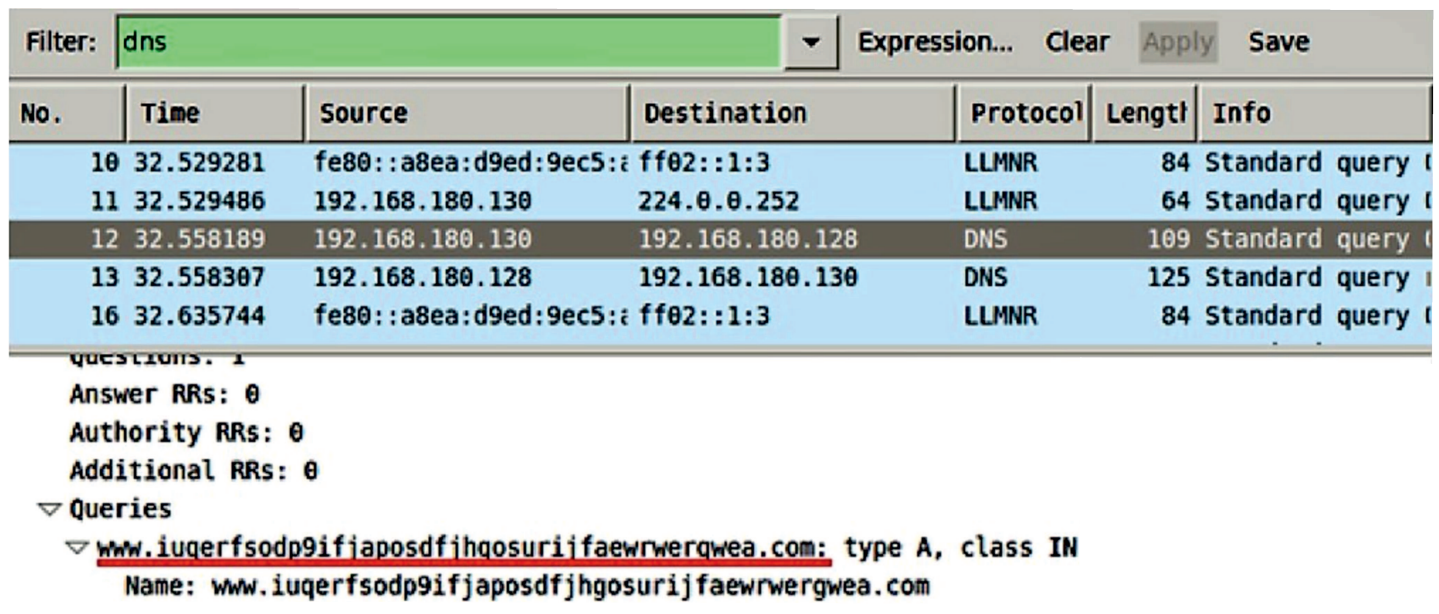

Fig. 3. Wireshark capture of the malicious DNS request.

\subsection{Initial Interactions}

The dynamic analysis conducted has revealed that, upon startup, the worm component tries to connect to the following domain, using the InternetOpenUrl function:

www.iuqerfsodp9ifjaposdfjhgosurijfaewrwergwea.com

The aforementioned domain is a kill-switch domain. This means that if the domain is active, the worm component stops running. On the other hand, if the worm component cannot establish a connection with this domain (e.g. if the domain is not active or if there is no connectivity), it continues to run and registers itself as a "Microsoft Security Center (2.0) Service" mssecsvs 2.0 process on the infected machine. Hence, this kill-switch domain may be used as part of a detection technique when developing a defense system.

The FakeDNS utility at REMnux captures the malicious DNS request on port 80 (Fig. 2), while Wireshark shows (Fig. 3) the DNS packet query field from the infected machine (IP 192.168.180.130) to the DNS server on REMnux (IP 192.168.180.128).

\subsection{Persistence Mechanisms}

After connection failure with the kill-switch domain, the worm component attempts to create a mssecsvs 2.0 process with the DisplayName of "Microsoft Security Center (2.0) Service". This can be observed in the Process Hacker tool with 4016 PID, indicating that the service has been launched (Fig. 4). In addition to this, the worm component of WannaCry extracts the hardcoded $R$ resource binary and then copies it to " $\mathrm{C}: \backslash$ Windows $\backslash$ taskche.exe" directory path. The $\mathrm{R}$ resource represents the binary of the WannaCry encryption component. After that, the worm runs the executable with the following parameters in the command line: "C: $\backslash$ Windows $\backslash$ taskche.exe/i". Next, the worm tries to move the " $\mathrm{C}: \backslash$ Windows $\backslash$ taskche.exe" file to "C: $\backslash$ Windows $\backslash$ qeriuwjhrf", to replace the original file if it exists. This is done to ensure multiple infections and avoid any issues with creating the tasksche.exe process.

\begin{tabular}{|l|l|}
\hline \hline Name & Display name \\
\hline$\Rightarrow$ mssecsvc2.0 & Microsoft Security Center (2.0) Service \\
\hline
\end{tabular}

Fig. 4. Microsoft Security Center (2.0) Service.

Finally, WannaCry creates an entry in the Windows registry in order to ensure that it runs every time the computer is restarted. The new entry contains a string (e.g. "midtxzggq900"), which is a unique identifier randomly generated by using the computer name. Once the tasksche.exe component runs, it copies itself to a folder with a randomly generated name in the Common Appdata directory of the infected machine. Then, it attempts to establish memory persistence by adding itself to the AutoRun feature. 
Created

Modifed $15 F 936$

Created

Modifed $30 \mathrm{C}$

Created

Created

Modifed

Modifed BBO7

Created

Modifed D457

Created

Modifed 135F2

Created

Modifed $989 \mathrm{E}$

Created

Modifed 9E40

Created

Modifed 90B5

Created

Modifed 907B

Created

Modifed 906D

Created

Modifed $92 \mathrm{CC}$

Created

Modifed 95E9
C: \ProgramData \midtxzggq900\b. wnry

c: \ProgramData \midtxzggq900 $\mathrm{\backslash b}$.wnry

C: \ProgramData\midtxzggq900\c. wnry

c: \ProgramData\midtxzggq900\c.wnry

C: $\backslash$ ProgramData $\backslash$ midtxzggq $900 \backslash \mathrm{msg}$

C: \ProgramData\midtxzggq900 \msg\m_bulgarian.wnry

C: $\backslash$ ProgramData $\backslash$ midtxzggq900 $\backslash \mathrm{msg}$

C: \ProgramData \midtxzggq900 \msg\m_bulgarian.wnry

C: \ProgramData \midtxzggg $900 \backslash \mathrm{msg} \backslash \mathrm{m}$ chinese (simplified). wnry

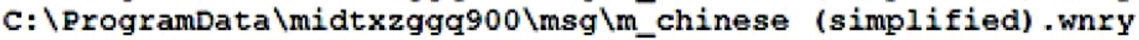

C: \ProgramData \midtxzggq900 $\mathrm{msg} \backslash \mathrm{m}$-chinese (traditional).wnry

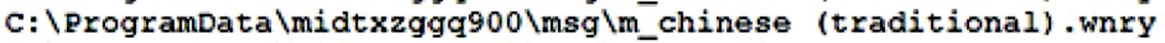

C: \ProgramData\midtxzggq $900 \backslash \mathrm{msg} \backslash \mathrm{m}$ croatian.wnry

C: \ProgramData \midtxzggq900\msg\m_croatian.wnry

C: \ProgramData \midtxzggq900\msg\m_czech.wnry

c: \ProgramData \midtxzggq900\msg\m_czech.wnry

C: \ProgramData \midtxzggq900\msg\m_danish.wnry

C: \ProgramData $\backslash$ midtxzggq $900 \backslash \mathrm{msg} \backslash \mathrm{m}$ _danish.wnry

C: \ProgramData \midtxzggq900\msg \m dutch. wnry

c: \ProgramData \midtxzggq $900 \backslash \mathrm{msg} \backslash \mathrm{m}$ _dutch.wnry

C: \ProgramData \midtxzggq900\msg\m_english.wnry

c: \ProgramData $\backslash$ midtxzggq900 $\backslash \mathrm{msg} \backslash \mathrm{m}$ _english.wnry

C: \ProgramData \midtxzggq900\msg\m filipino.wnry

C: \ProgramData \midtxzggq $900 \backslash \mathrm{msg} \backslash \mathrm{m}$-filipino.wnry

C: \ProgramData \midtxzggq $900 \backslash \mathrm{msg} \backslash \mathrm{m}$ finnish.wnry

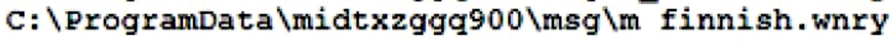

Fig. 5. WannaCry dropped files to the working directory.

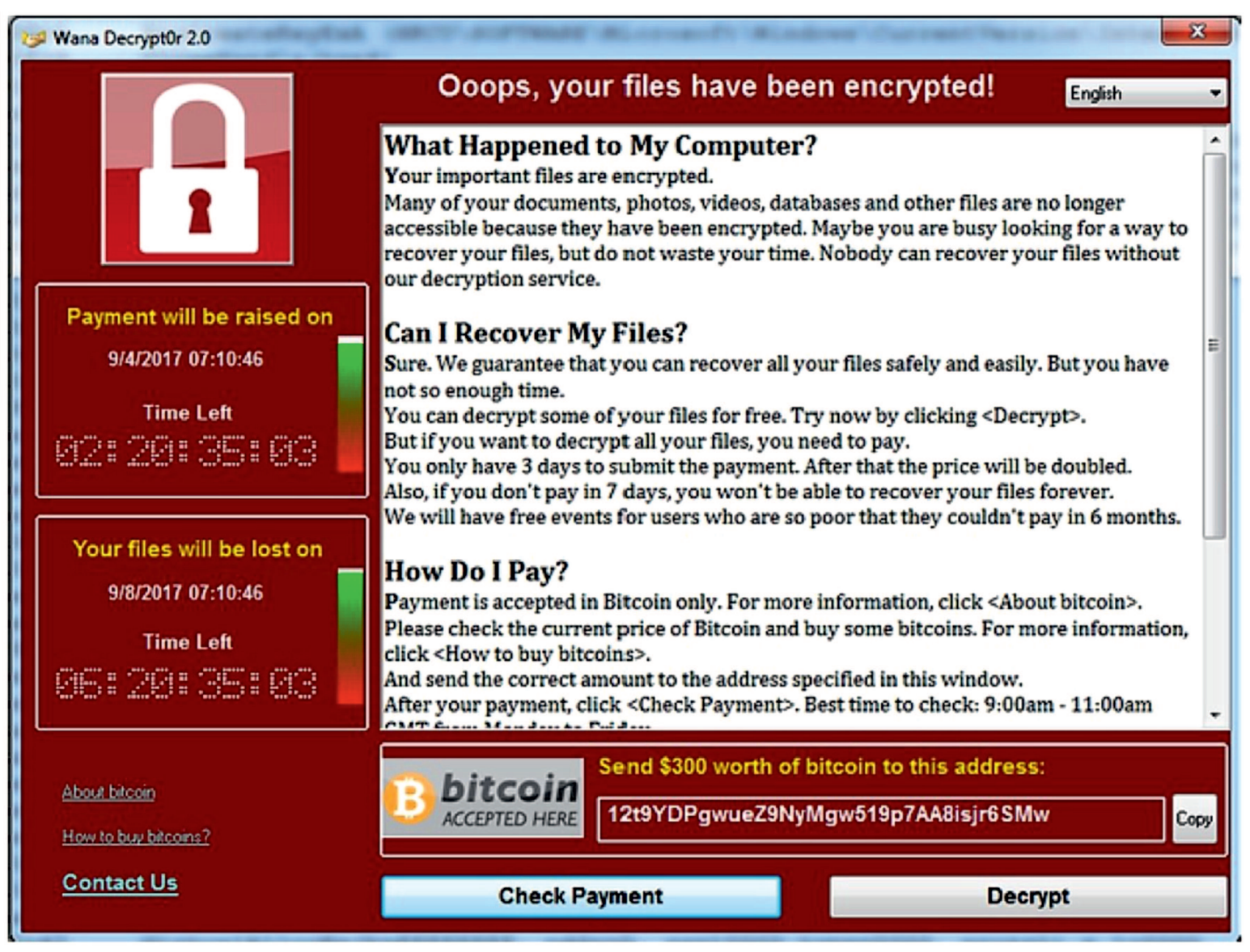

Fig. 6. WannaCry extortion message. 
In summary, the dynamic analysis has revealed that, to achieve persistence on the infected machine, WannaCry performs the following actions:

- creates an entry in the Windows registry to ensure that it executes every time the machine is restarted,

- attempts to achieve memory persistence by adding itself to the AutoRun feature of Windows,

- uses Windows icacls command to grant itself a full access to all files on the machine,

- deletes all backup (shadow) copies and tries to prevent being booted in safe mode by executing several commands in the Windows command line,

- deletes all backup folders,

- by using the Windows command line, creates a VBScript program which generates a single shortcut of the@WanaDecryptor@.exe decrypter file,

- tries to kill SQL and MS Exchange database processes by executing several commands in the Windows command line.

\subsection{Configuration Data Load}

After the persistence phase, WannaCry loads the XIA resource, which corresponds to a password protected ZIP file. It decompresses the files and drops them to the working directory of the running process (Fig. 5), as observed in the DirWatch module of SysAnalyzer.

As one can see, WannaCry loads configuration data from the c.wnry file into memory. WannaCry randomly chooses one of the three available Bitcoin addresses and then writes this address back to the configuration data. This is done in order to display the payment address in the extortion message (Fig. 6). After that, WannaCry sets the hidden attribute (Fig. 7) for the working directory with the help of the CreateProcess function. Next, with the help of the Windows icacls command, WannaCry grants full access to all files on the target system (Fig. 8).

\begin{tabular}{|l|l|l|}
\hline PID & User & CmdLine \\
\hline F5C & SYSTEM & attrib +h. \\
\hline
\end{tabular}

Fig. 7. WannaCry sets the hidden attribute for the working directory.

\begin{tabular}{l|l|l}
\hline PID & User & CmdLine \\
\hline D14 & SYSTEM & icacls . /grant Everyone:F /T /C /Q \\
\hline
\end{tabular}

Fig. 8. WannaCry grants full access on the target system.

The next step is to import one of the hardcoded public RSA keys as was identified at offset 0xec00 of the tasksche.exe process (Fig. 9). WannaCry then loads and executes, in memory, the contents of the t.wnry file (Fig. 10) which contains the default encrypted AES key required for decrypting the DLL responsible for the file encryption routine. The first 8 bytes of the file are checked to match the WANACRY! string. Then, the imported public RSA key hardcoded within binary is used to decrypt the AES key stored at the beginning of the t.wnry file. The AES key obtained is then used to decrypt and load the encryption DLL, which can be observed with the help of OllyDbg debugging tool [17] during WannaCry execution, as shown in Fig. 11. This DLL is responsible for file encryption on the infected machine and is summarized in Table 6.

Table 6

Encryption DLL

\begin{tabular}{|c|l|}
\hline MD5 & f351e1fcca0c4ea05fc44d15a17f8b36 \\
\hline SHA1 & $\begin{array}{l}\text { 7d36a6aa8cb6b504ee9213c200c831e } \\
\text { b8d4ef26b }\end{array}$ \\
\hline Size & 65536 bytes \\
\hline File type & Dynamic-Link-Library \\
\hline Internal name & kbdlv.dll \\
\hline File description & Latvia keyboard layout \\
\hline Timestamp & Mon, Jul 13 18:12:55 2009 \\
\hline
\end{tabular}

\subsection{Encryption Process}

The encryption component of WannaCry is invoked with the TaskStart system thread. During its execution, the encryption component checks if one of the following mutexes exists:

\section{GlobalnMsWinZonesCacheCounterMutexA, GlobalnMsWinZonesCacheCounterMutexW, MsWinZonesCacheCounterMutexA.}

If the mutex "MsWinZonesCacheCounterMutexA" is present, then the encryption component automatically stops without taking any further action. If the mutex is not present on the system, the encryption process starts. In particular, TaskStart creates a new mutex named "MsWinZonesCacheCounterMutexA" and reads the contents of the c.wnry file from the current directory. After that, WannaCry creates three configuration files shown in Table 7.

Table 7

WannaCry configuration files

\begin{tabular}{|c|l|}
\hline Filename & \multicolumn{1}{|c|}{ Description } \\
\hline \hline 00000000.res & TOR/C2 info \\
\hline 00000000.pky & Public RSA key \\
\hline 00000000.eky & Encrypted private RSA key \\
\hline
\end{tabular}

After the configuration files have been created, the encryption component is ready to start encrypting files on the system. To accomplish this, it spawns several threads. First, 


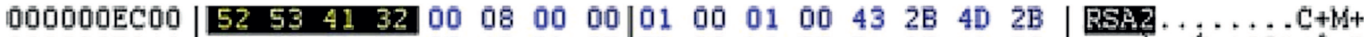

1000000EC10 04 9C 0A D9 9F 1E DA 5F ED 32 A9 EF E1 CE 1 A 50 ..Û.,U_i201á1.P

$000000 \mathrm{EC} 20$ F4 15 E7 51 7B EC BO $27 \mid 56$ 05 58 B4 F6 83 C9 B6

Fig. 9. Imported RSA private key.

\begin{tabular}{|c|c|c|c|c|c|c|c|c|c|c|c|c|c|c|c|c|c|}
\hline \multicolumn{18}{|l|}{ t.wnry } \\
\hline Offset & 0 & 1 & 2 & 3 & 4 & 5 & 6 & 7 & 8 & 9 & A & B & C & $\mathrm{D}$ & E & $\mathrm{F}$ & ANSI ASCII \\
\hline 00000000 & 57 & 41 & $4 \mathrm{E}$ & 41 & 43 & 52 & 59 & 21 & 00 & 01 & 00 & 00 & $1 \mathrm{E}$ & 38 & 22 & 27 & WANACRY! \\
\hline 00000010 & FD & E6 & $7 \mathrm{~F}$ & oc & $5 \mathrm{D}$ & E7 & $7 \mathrm{E}$ & $3 E$ & 28 & A.7 & $\mathrm{AF}$ & FD & $2 \mathrm{~A}$ & 50 & 64 & 49 & \multirow{3}{*}{ 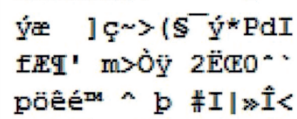 } \\
\hline 00000020 & 66 & $\mathrm{c} 6$ & B6 & 27 & 17 & $6 \mathrm{D}$ & $3 \mathrm{E}$ & $\mathrm{D} 2$ & $\mathrm{FF}$ & $1 \mathrm{C}$ & 32 & $\mathrm{CB}$ & $8 \mathrm{C}$ & 30 & 88 & 60 & \\
\hline 00000030 & 70 & F6 & $\mathrm{EA}$ & E9 & 99 & 81 & $5 \mathrm{E}$ & 15 & $\mathrm{FE}$ & 03 & 23 & 49 & $7 \mathrm{C}$ & BB & $\mathrm{CE}$ & $3 \mathrm{C}$ & \\
\hline 00000040 & EE & 57 & EO & 42 & $\mathrm{DC}$ & $3 \mathrm{D}$ & $\mathrm{AF}$ & $\mathrm{A} 8$ & 82 & $\mathrm{~B} 8$ & $4 \mathrm{D}$ & 01 & 05 & $7 \mathrm{~A}$ & 78 & 46 & îWàBÜ $=-.,, M \quad$ zxF \\
\hline 00000050 & 70 & $\mathrm{OE}$ & $\mathrm{A} 8$ & $\mathrm{DD}$ & E5 & 30 & 65 & B5 & B1 & F1 & 50 & $\mathrm{EE}$ & 10 & $1 \mathrm{D}$ & B3 & 22 & 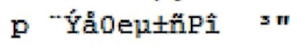 \\
\hline
\end{tabular}

Fig. 10. Loaded and executed t.wnry file.

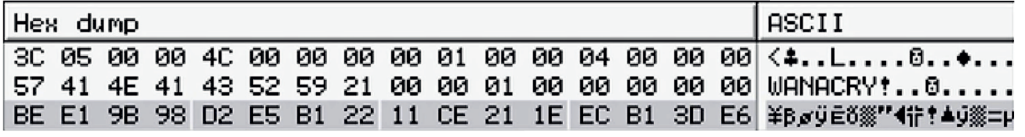

Fig. 11. Decrypted AES key in a memory dump.

WannaCry attempts to load and check the existence of two keys in the 00000000.pky and 00000000.dky files. The 00000000.dky file presents a decryption RSA key which is received upon the payment has been verified. When the victim clicks the "Check Payment" button, WannaCry starts checking for the presence of the 00000000.dky file on the system. If the two aforementioned files do not exist, WannaCry generates a new unique RSA 2048-bit asymmetric key pair, which can be seen in the memory dump made with with SysAnalyzer tool at 0x2B3795 offset (Fig. 12).

\begin{tabular}{|l|l|}
\hline Offset & Data \\
\hline 2B3795 & generating RSA key \\
\hline
\end{tabular}

Fig. 12. Generation of an RSA key pair.

Once the key pair has been generated, WannaCry exports the victim's public RSA key to a 00000000.pky file using Microsoft's CryptExportKey function. Next, WannaCry exports the victim's private RSA key and encrypts it with another hard-coded RSA public key. The encrypted private key is stored as a 00000000.eky file. After the key has been safely stored, WannaCry calls upon the CryptDestroyKey function to destroy the private key in memory, to limit any key recovery options.

Next, WannaCry starts enumerating, every 3 seconds, information about all logical drives attached to the system. If a new attached drive is not a CD ROM drive, then it begins the encryption process on the new drive. At this stage, WannaCry also starts iterating through all existing directories and searching for predefined file extensions of interest.
To encrypt each file, it generates a 16-byte symmetric AES key using the CryptGenRandom function. Then, it encrypts every generated AES key with the public RSA key and stores it inside the file header starting with the WANACRY! string value. Encrypted files are renamed and appended with the .WNCRY file extension.

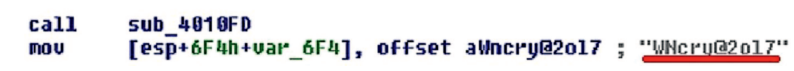

Fig. 13. Password for a ZIP archive in the encryption component.

The encryption component contains a password-protected ZIP archive. We managed to obtain the password, "WNcry@2ol7", by disassembling the encrypter with the IDA Pro tool [18] (see Fig. 13). The contents of the ZIP archive are summarized in Table 8 and described below:

- $m s g$ is a folder that contains a list of rich text format (RTF) files with the wnry extension. These files are the readme instructions used to show the extortion message to the victim in different languages, based on the information obtained from the system by malicious WannaCry functions;

- b.wnry is an image file used for displaying instructions for the decryption of user files. It starts with 42 4D strings, which indicates that this file is a bitmap image;

- c.wnry contains a list of Tor addresses with the .onion extension and a link to a zipped installation file of the Tor browser from Tor Project [19]; 
Table 8

Files in the password protected ZIP archive

\begin{tabular}{|c|c|c|}
\hline Name & Size [bytes] & Modified \\
\hline \hline msg & $1,329,657$ & $2017-05-11$ \\
\hline b.wnry & $1,440,054$ & $2017-05-11$ \\
\hline c.wnry & 780 & $2017-05-11$ \\
\hline r.wnry & 864 & $2017-05-10$ \\
\hline s.wnry & $3,038,286$ & $2017-05-09$ \\
\hline t.wnry & 65,816 & $2017-05-11$ \\
\hline taskdl.exe & 20,480 & $2017-05-11$ \\
\hline taskse.exe & 20,480 & $2017-05-11$ \\
\hline u.wnry & 245,760 & $2017-05-11$ \\
\hline
\end{tabular}

- r.wnry is a text file in English with additional decryption instructions to be used by the decryption component (the u.wnry file mentioned below);

- s.wnry file is a ZIP archive (HEX signature 50 4B 03 04) which contains the Tor software executable. This executable has been obtained with the assistance of the WinHex tool [20] by saving raw binary data with the .zip extension;

- t.wnry is an encrypted file with the WANACRY! encryption format. The file header starts with the WANACRY! string;

- taskdl.exe is a supporting tool for the deletion of files with the .WNCRY extension. By observing the properties of the file, the following masquerade description can be found: "SQL Client Configuration Utility";

- taskse.exe is a supporting tool for malware execution on remote desktop protocol (RDP) sessions. The following file description was identified: "waitfor wait/send a signal over a network";

- u.wnry is an executable file (HEX signature 4D 5A) with the name of “@WanaDecryptor@.exe”, which represents the decryption component of WannaCry.

At the same time, another thread calls the taskse.exe process every $30 \mathrm{~s}$, which tries to enumerate active RDP sessions on connected remote machines and to run the @WanaDecryptor@.exe binary file. This file is extracted from the u.wnry file and represents the decryption component of WannaCry. The persistence of RDP session injections is ensured by adding the value in the AutoRun registry key.

\subsection{Recovery Prevention}

After finishing the encryption process, WannaCry tries to prevent various common data recovery methods by executing several commands on the system. To prevent data recovery, WannaCry executes the following commands:

- vssadmin delete shadows/all/quiet. Deletes all the shadow volumes on the system without alerting the user. By default, these volumes contain backup data in the event of a system fault;

- wmic shadowcopy delete. Ensures deletion of any copies relevant to shadow volumes;

- bcdedit/set default bootstatuspolicy ignoreallfailures. Ensures that the machine is booted, even if errors are found;

- bcdedit/set default recoveryenabled no. Disables the Windows recovery feature, thus preventing the victims from the possibility to reverting their system to a previous build;

- wbadmin delete catalog $-q$. Ensures that victim can no longer use any backup files created by Windows Server.

\subsection{Propagation}

The worm component of WannaCry carries the main propagation and exploit functionality, which utilizes the EternalBlue exploit and the DoublePulsar backdoor to leverage the MS17-010 SMB vulnerability [12]. After performing the initial interactions and checking connectivity with the kill-switch domain, the worm functionality is established by initiating the mssecsvs 2.0 service, which WannaCry installs after being executed. This service tries to spread WannaCry payload through the SMB vulnerability on any vulnerable systems on both internal and external networks.

In order to perform this, WannaCry creates and spawns two separate threads that simultaneously replicate worm payload in all detected networks. In the internal network, before starting the propagation process, the component obtains the IP addresses of local network interfaces by invoking the GetAdaptersInfo function, and determines the subnets existing in the network.

After that, the worm component tries to connect to all possible IP addresses in any available local network on port 445, which is the default port for SMB over IP service. If successful, the worm attempts to exploit the service for the MS17-010 vulnerability. In our testbed, connection attempts were observed with Wireshark on a REMnux machine, when the infected machine (IP 192.168.180.130) sent SMB probe packets to the clean machine (IP 192.168.180.134), as shown in Fig. 14.

During the SMB probing, one of the unique features of the generated traffic is that it contains two hardcoded IP addresses: 192.168.56.20 and 172.16.99.5. They can be observed by extracting strings from the binary. In particular, WannaCry sends three NetBIOS session setup packets, where two of them contain the aforementioned hardcoded IP addresses.

At the same time, the worm component attempts to spread across the external networks by generating various IP addresses and by trying to connect to TCP port 445. This can be observed with Wireshark on REMnux, as shown 


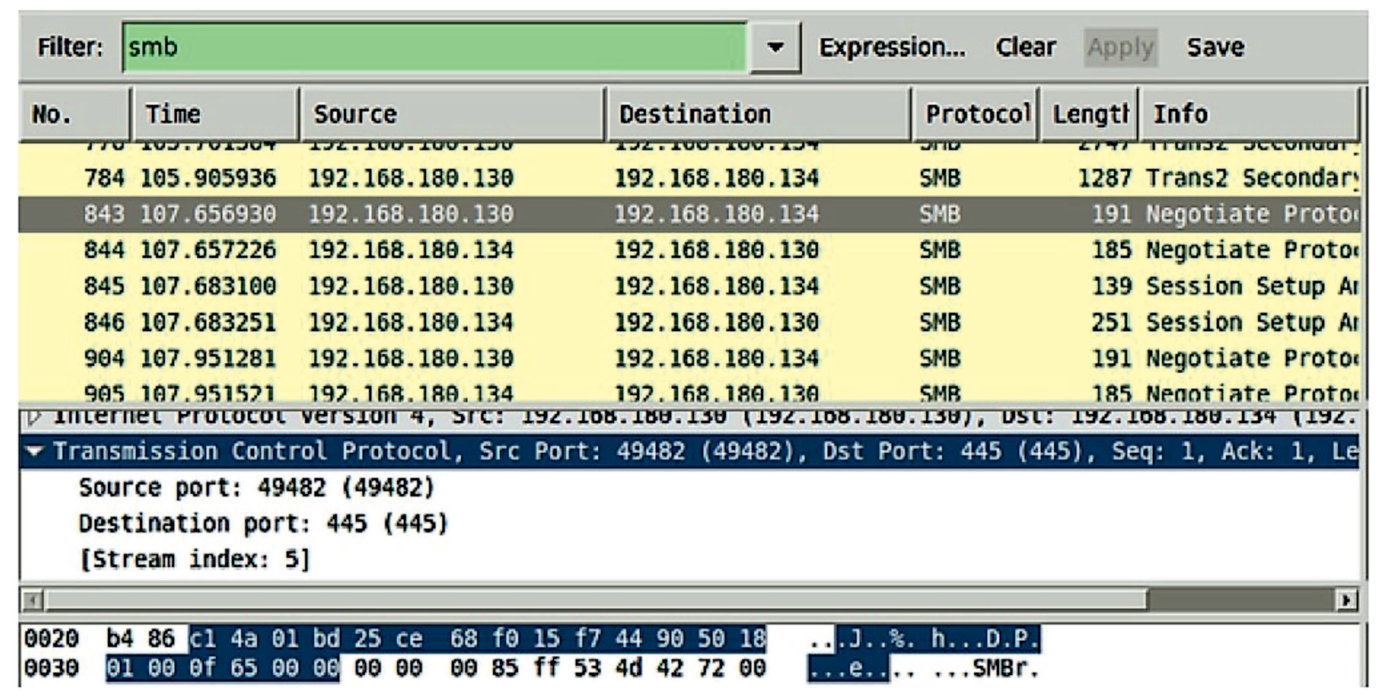

Fig. 14. WannaCry internal network traffic attempting the SMB exploit.

\begin{tabular}{|c|c|c|c|c|c|c|}
\hline \multicolumn{4}{|c|}{ Filter: tcp } & - Expression... & \multicolumn{2}{|c|}{ Apply Save } \\
\hline No. & Time & Source & Destination & Protocol & Lengtl & Info \\
\hline $115 \theta$ & 76.598100 & $192.168 .180 .13 \theta$ & 109.140 .223 .210 & TCP & 66 & $4977 \theta>445[\mathrm{SY}$ \\
\hline 1151 & 76.598259 & $192.168 .180 .13 \theta$ & 206.242 .244 .156 & TCP & 66 & $49771>445[\mathrm{SY}]$ \\
\hline 1152 & 76.598308 & $192.168 .180 .13 \theta$ & $52.213 .9 \theta .24 \theta$ & TCP & 66 & $49772>445[\mathrm{SY}]$ \\
\hline 1153 & 76.598386 & $192.168 .180 .13 \theta$ & 202.76 .26 .154 & TCP & 66 & $49773>445[\mathrm{SY}$ \\
\hline 1154 & 76.598466 & $192.168 .180 .13 \theta$ & 205.215 .5 .24 & TCP & 66 & $49774>445[\mathrm{SY}]$ \\
\hline 1155 & 76.598549 & $192.168 .180 .13 \theta$ & 80.133 .73 .130 & TCP & 66 & $49775>445$ [SY] \\
\hline 1156 & $76.5987 \theta 8$ & 192.168 .180 .130 & 198.73 .58 .205 & TCP & 66 & $49776>445$ [SY] \\
\hline 1157 & 76.931700 & $192.168 .18 \theta .13 \theta$ & 40.188 .28 .244 & TCP & 66 & $49779>445[\mathrm{SY}$ \\
\hline 1158 & 76.931759 & $192.168 .180 .13 \theta$ & 184.55 .110 .103 & TCP & 66 & $4978 \theta>445[\mathrm{SY}$ \\
\hline
\end{tabular}

Fig. 15. WannaCry external network traffic attempting the SMB exploit.

in Fig. 15. As it can be seen, the worm attempts to probe external Internet IP addresses for the MS17-010 vulnerability. This explains the reason for the widespread infec-

Table 9

External IP addresses generated by WannaCry

\begin{tabular}{|c|}
\hline IP address : port \\
\hline $109.140 .223 .210: 445$ \\
\hline $206.242 .244 .156: 445$ \\
\hline $52.213 .90 .240: 445$ \\
\hline $202.76 .26 .154: 445$ \\
\hline $205.215 .5 .24: 445$ \\
\hline $80.133 .73 .130: 445$ \\
\hline $198.73 .58 .205: 445$ \\
\hline $40.188 .28 .244: 445$ \\
\hline $184.55 .110 .103: 445$ \\
\hline
\end{tabular}

tion seen during the massive outbreak on 12 May 2017. The full list of WannaCry generated IP addresses obtained during the analysis is presented in Table 9.

\section{9. $C \& C$ Communication}

During its execution, the software also tries to contact the C\&C servers. To this end, WannaCry unpacked and dropped files from the s.wnry file, containing the Tor executable, into the installation directory as shown

$$
\begin{array}{ll}
\text { Created } & \text { C: } \backslash \text { ProgramData } \backslash \text { midtxzggq900\s.wnry } \\
\text { Modifed 2E5C4E } & \text { C: } \backslash \text { ProgramData \midtxzggq900\s.wnry }
\end{array}
$$

Fig. 16. Tor executable dropped into the installation directory.

in Fig. 16. Before unpacking, it starts listening on the localhost address 127.0.0.1:9050. This address, with the specified 9050 port, is typically used for configuring the 
Tor browser application. If the contents of the s.wnry file are corrupted, then WannaCry tries to download the Tor executable from a hardcoded URL. After the successful extraction of the Tor executable, it copies "TaskData $\backslash$ Tor $\backslash$ tor.exe" to "TaskData $\backslash$ Tor $\backslash$ taskhsvc.exe" and executes it. Next, WannaCry parses the contents of the c.wnry file, which specifies the configuration data, including the following .onion addresses to connect and the zipped Tor browser installation file:

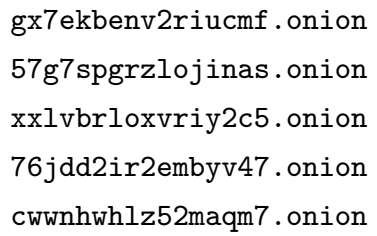

After that, WannaCry sends the first eight bytes of the 00000000.res file content to the C\&C server. These bytes specify the host and user name of the infected machine. The 00000000.res file, which is dropped during encryption process, accumulates in total 88 bytes of configuration data, including internal flags, counters, and timestamps.

During its communication with Tor addresses, WannaCry establishes a secure HTTPS channel to port 443, and uses common Tor ports, 9001 and 9050, for network traffic and directory information.

\section{Conclusions and Future Work}

We have performed a comprehensive dynamic analysis of WannaCry ransomware in a purpose-built virtual testbed. We analyzed the WannaCry version which was observed during the massive attacks on 12 May 2017. The analysis has revealed that the given ransomware is composed of two distinctive components, which enable the worm-like selfpropagating mechanism and combined encryption process. Both worm and encryption components of WannaCry have been examined.

The focus of this study was on WannaCry's initial interactions and the infection process, its persistence mechanism, encryption process, recovery prevention as well as its propagation mechanisms and communication with $\mathrm{C} \& \mathrm{C}$ servers. The analysis has revealed important characteristics and behaviors of WannaCry during its execution. In particular, we identified Tor addresses used for C\&C, observed TCP and DNS connections, SMB probes, as well as actions related to WannaCry persistence and obfuscation.

The worm component of WannaCry weaponized by the functionality enabling it to exploit and propagate via Microsoft's MS17-010 on unpatched systems by sending SMB probing packets on port 445. In addition to the modular nature of WannaCry, it was also observed that it has embedded RSA keys used to decrypt the required malicious DLL representing the encryption component. It was identified that the worm component scans both internal and external networks for MS17-010 vulnerability, by generating a list of local and global IP addresses. The worm tries to probe the hosts from the generated list by sending packets to port 445. Before its execution, WannaCry also performs an initial check with the kill-switch domain.

At the same time, the analysis has identified two hardcoded IP addresses (192.168.56.20 and 172.16.99.5), which are sent during the SMB probing. Depending on the condition of the s.wnry file dropped during execution, WannaCry can also communicate with embedded .onion addresses via a secure channel on port 443 and via common Tor ports 900 and 9050 to download the Tor browser installation software from a specified URL.

The findings of this work could be used for designing effective mitigation mechanisms for WannaCry and other ransomware families that exhibit similar behavior. This is left as future work. In particular, we plan to investigate the use of software-defining networking (SDN) [21], [22] for ransomware detection and mitigation. SDN is an emerging paradigm of programmable networks that decouples the control and data planes. SDN controllers maintain a view of the entire network and implement policy decisions. On the other hand, each device at the data plane maintains one or more flow tables, where the packet handling rules are stored. This changes the way that networks are designed and managed, and enables new SDN-based security solutions [23]-[25], such as firewalls and intrusion detection systems for various types of malware, including ransomware mitigation [26], [27].

\section{References}

[1] D. O'Brien, "Ransomware 2017", Internet Security Threat Report, Symantec, July 2017 [Online]. Available: https://www.symantec.com/content/dam/symantec/docs/securitycenter/white-papers/istr-ransomware-2017-en.pdf

[2] K. Savage, P. Coogan, and H. Lau, "The evolution of ransomware", Security Response, Symantec, June 2015 [Online]. Available: http://www.symantec.com/content/en/us/enterprise/ media/security_response/whitepapers/the-evolution-ofransomware.pdf

[3] A. Zeichnick, "Self-propagating ransomware: What the WannaCry ransomworm means for you", May 2017 [Online]. Available: https://www.networkworld.com/article/3196993/security/selfpropagating-ransomware-what-the-wannacry-ransomworm-meansfor-you.html

[4] "Ransom.Wannacry", Symantec, May 2017 [Online]. Available: https://www.symantec.com/security-center/writeup/2017-0513103522-99/

[5] "Petya - taking ransomware to the low level", Malwarebytes Labs, Jun. 2017 [Online]. Available: https://blog.malwarebytes.com/ threat-analysis/2016/04/petya-ransomware/

[6] "Petya ransomware eats your hard drives", Kaspersky Labs, Jun. 2017 [Online]. Available: https://www.kaspersky.com/blog/ petya-ransomware/11715 
[7] "Bad Rabbit: A new ransomware epidemic is on the rise", Kaspersky Labs, Oct. 2017 [Online]. Available: https://www.kaspersky.com/ blog/bad-rabbit-ransomware/19887/

[8] M. Akbanov, V. G. Vassilakis, I. D. Moscholios, and M. D. Logothetis, "Static and dynamic analysis of WannaCry ransmware", in Proc. IEICE Inform. and Commun. Technol. Forum ICTF 2018, Graz, Austria, 2018.

[9] C. Everett, "Ransomware: To pay or not to pay?", Comp. Fraud \& Secur., vol. 2016, no. 4, pp. 8-12, 2016 (doi: 10.1016/S1361-3723(16)30036-7).

[10] "Understanding ransomware and strategies to defeat it", McAfee Labs, White Paper, 2016 [Online]. Available: https://www.mcafee.com/enterprise/en-us/assets/white-papers/ wp-understanding-ransomware-strategies-defeat.pdf

[11] "What you need to know about the WannaCry ransomware", Symantec, Threat Intelligence, Oct. 2017, [Online]. Available: https://www.symantec.com/blogs/threat-intelligence/wannacryransomware-attack

[12] Microsoft Security Bulletin MS17-010 - Critical, March 14, 2017 [Online]. Available: https://docs.microsoft.com/en-us/ security-updates/securitybulletins/2017/ms17-010

[13] ViRus Share malware repository [Online]. Available: https://virusshare.com (accessed Nov. 30, 2018).

[14] "REMnux: A Linux toolkit for reverse-engineering and analyzing malware" [Online]. Available: https://remnux.org (accessed Nov. 30, 2018).

[15] SysAnalyzer - Automated malcode analysis system [Online]. Available: https://github.com/dzzie/SysAnalyzer (accessed Nov. 30, 2018).

[16] Pestudio, Malware Assessment Tool [Online]. Available: https://www.winitor.com (accessed Nov. 30, 2018).

[17] OllyDbg - A 32-bit assembler level debugger for Microsoft Windows [Online]. Available: http://www.ollydbg.de/ (accessed Nov. 30, 2018).

[18] IDA: Pro [Online]. Available: https://www.hex-rays.com/ products/ida (accessed Nov. 30, 2018).

[19] Tor Project [Online]. Available: https://www.torproject.org (accessed Nov. 30, 2018).

[20] "WinHex: Computer forensics and data recovery software" [Online]. Available: https://www.X-ways.net/winhex (accessed Nov. 30, 2018).

[21] B. Nunes, M. Mendonca, X. N. Nguyen, K. Obraczka, and T. Turletti, "A survey of software-defined networking: Past, present, future of programmable networks", IEEE Commun. Surveys \& Tutor., vol. 16, no. 3 , pp. $1617-1634,2014$ (doi: 10.1109/SURV.2014.012214.00180)

[22] V. G. Vassilakis, I. D. Moscholios, B. A. Alzahrani, and M. D. Logothetis, "A software-defined architecture for next-generation cellular networks", in Proc. IEEE Int. Conf. on Commun. ICC 2016, Kuala Lumpur, Malaysia, 2016 (doi: 10.1109/ICC.2016.7511018).

[23] C. Yoon, T. Park, S. Lee, H. Kang, S. Shin, and Z. Zhang, "Enabling security functions with SDN: A feasibility study", Comp. Netw., vol. 85, pp. 19-35, 2015 (doi: 10.1016/j.comnet.2015.05.005).

[24] J. M. Ceron, C. B. Margi, and L. Z. Granville, "MARS: An SDNbased malware analysis solution", Proc. IEEE Symp. on Comp. and Commun. ISCC 2016, Messina, Italy, 2016 (doi: 10.1109/ISCC.2016.7543792)

[25] V. G. Vassilakis, I. D. Moscholios, B. A. Alzahrani, and M. D. Logothetis, "On the security of software-defined next-generation cellular networks", in Proc. IEICE Inform. and Commun. Technol. Forum ICTF 2016, Patras, Greece, 2016.

[26] K. Cabaj and W. Mazurczyk, "Using software-defined networking for ransomware mitigation: The case of CryptoWall", IEEE Network, vol. 30 , no. 6 , pp. 14-20, 2016 (doi: 10.1109/MNET.2016.1600110NM).
[27] K. Cabaj, M. Gregorczyk, and W. Mazurczyk, "Software-defined networking-based crypto ransomware detection using HTTP traffic characteristics", Comp. \& Elec. Engin., vol. 66, pp. 353-386, 2018 (doi: 10.1016/j.compeleceng.2017.10.012).

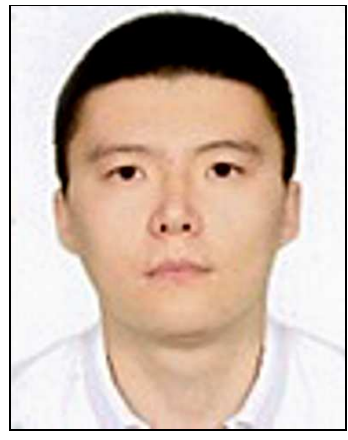

Maxat Akbanov received the B.Sc. degree in Information and Communications System Security from the National Technical University of Ukraine "Kyiv Polytechnic University", Kyiv, Ukraine, in 2011, and the M.Sc. degree in Cyber Security from the University of York, York, UK, in 2018. In 2008 and 2016, he received the prestigious Kazakhstan governmental "Bolashak" scholarship to fund his studies abroad. He holds merit and distinction awards for B.Sc. and M.Sc. degrees, respectively. He is currently working for the private sector in Kazakhstan and is involved in developing several startup projects for the government-sponsored "Digital Kazakhstan" and "Cyber Shield" strategies. His main research interests include network and malware forensics, software-defined networking, covert channels, cryptography, Internet of Things, machine learning and artificial intelligence.

E-mail: maxat.akbanov@gmail.com

Department of Computer Science

University of York

Deramore Lane

Heslington

York YO10 5GH, United Kingdom

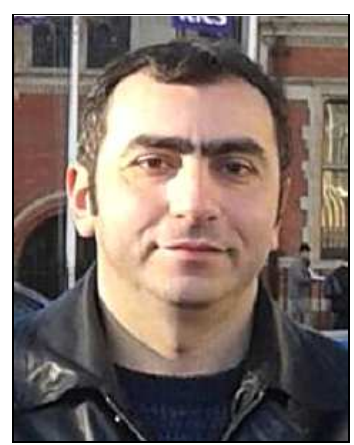

Vassilios G. Vassilakis received his Ph.D. degree in Electrical and Computer Engineering from the University of Patras, Greece in 2011. He is currently a lecturer in Cyber Security at the University of York, UK. He's been involved in EU, $\mathrm{UK}$, and industry funded R\&D projects related to the design and analysis of future mobile networks and Internet technologies. His main research interests are in the areas of network security, Internet of Things, next-generation wireless and mobile networks, and software-defined networks. He has published over 90 papers in international journals/conferences. He has served as a Guest Editor in IEICE Transactions on Communications, IET Networks, and Elsevier Optical Switching $\&$ Networking, and in the TPC of IEEE ICC and IEEE Globecom.

E-mail:vv274@cl.cam.ac.uk

University of York

York YO10 5DD, United Kingdom 


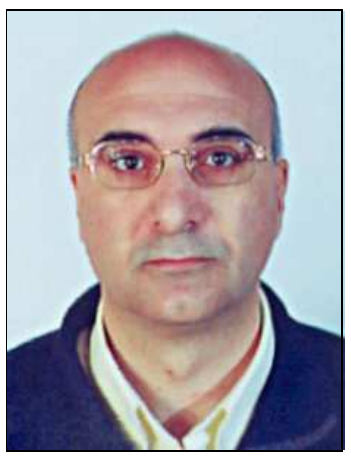

Michael D. Logothetis received his B.Eng. degree and $\mathrm{Ph} . \mathrm{D}$. in Electrical Engineering, both from the University of Patras, Patras, Greece, in 1981 and 1990, respectively. From 1991 to 1992 he was a Research Associate at NTT's Telecommunication Networks Laboratories, Tokyo, Japan. In 2009 he was elected (Full) Professor at the ECE Department of the University of $\mathrm{Pa}$ tras. His research interests include teletraffic theory, simulation and performance optimization of telecommuni- cations networks. He has published over 200 conference/journal papers. He has become a Guest Editor in: Mediterranean Journal of Electronics and Communications, Mediterranean Journal of Computers and Networks, IET Circuits, Devices and Systems, IET Networks and Ubiquitous Computing and Communication Journal. He is a member of the IARIA (Fellow), IEEE (Senior), IEICE (Senior), FITCE and the Technical Chamber of Greece (TEE).

E-mail: mlogo@upatras.gr

Wire Communications Laboratory

Department of Electrical and Computer Engineering University of Patras

26504 Patras, Greece 